\title{
Human Emotions and Physiological Signals: A Classroom Experiment
}

\author{
http://dx.doi.org/10.3991/ijoe.v12i04.5098
}

\author{
Bruno Patrão, Samuel Pedro and Paulo Menezes \\ University of Coimbra, Portugal
}

\begin{abstract}
In this paper we present a Virtual Reality based laboratory experience that can be used to demonstrate the effect that emotions may play in our bodies. For attaining this purpose, a Virtual Reality-based system is presented where three different virtual environments aim at inducing specific sensations and emotions on the students participating in a classroom experiment. The objective is that the students be able to analyze their own physiological data and understand the correlation between data patterns and experienced situation.
\end{abstract}

Index Termss-Student Experiments, Biomedical Engineering Education, Virtual Reality, Emotion Induction

\section{INTRODUCTION}

In this paper we present a Virtual Reality (VR) based laboratory experience that enables K12 students to understand the effect that emotions play in our bodies. The signs of emotional arousal involve changes in the activity of the autonomic system that is regulated in part by circuits in the cerebral cortex, such as, involuntary reactions like blushing in response to surround embarrassing stimuli or vasoconstriction and pallor in response to fear. Moreover, autonomic function is closely related to emotional experience and expression. Thus, increases or de- creases in heart rate, cutaneous blood flow (blushing or turning pale) and sweating can all accompany various emotions. These responses are delivered by activity changes in the sympathetic and parasympathetic system, which govern smooth muscle, cardiac muscle, and glands in every part of the body [1]. There are some studies conducted in the field of bio-signals acquisition and data analysis in order to recognize emotions and/or improve the humancomputer interaction [2], [3]. Other authors defend the idea that virtual reality, immersive and affective systems can be a powerful tool for learning especially when the targets are young students [4], [5], in part due to their good acceptance, given some similarities to video games. The introduction of low-cost immersive devices and systems capable of taking a user into a totally different space, by tricking his/her senses, creates an excellent opportunity for manipulating his/her emotions. These new affective interfaces can be coupled with physiological sensors, creating a system able to not only induce emotional states, but also analyze (and eventually react) their physiological responses [6].

\section{EXPLOITING AFFECTIVE INTERACTION TO INDUCE Measurable Physiological Changes}

The recent rise of head mounted displays is making virtual reality environments more immersive, affordable and easy to use. These devices allow the user to be in a variety of believable scenarios removing any risk of injury while still keeping the sense of presence. The head mounted display completely deceives the user's vision sense but, to make him/her feel truly immersed, this visual experience has to be accompanied with sound. Sound plays an important role in all of the created virtual environments and it's an integral part of any virtual reality experience even more so than realistic graphics [7]. This ability to make a user feel like he/she is in an environment different than the real one opens up many possibilities. Negative emotions like stress, fear and anxiety and positive emotions like relaxation, calm and tranquility can be accurately elicited [8]. This opens a new possibility of exploring the use of VR to produce sensations and induce emotional states. In this work we propose the exploitation of this to teach the students about how the emotions may produce changes in the physiological signals.

\section{A. Using Virtual Reality to Induce Emotions}

With the purpose of demonstrating the dependency of the physiological measurable parameters on the mental state, we propose the use of a laboratory experiment as will be described later in this article. This has two parts, one immersive and stimulating part for guiding the student in performing the experiment and the second part devoted to bio-signals acquisition, pre-processing and delivery. We will concentrate essentially on the first part, as for the second part we will only discuss the collected data and analyze the results.

Considering that students already understand the relationship between heart rate variations and physical activities, the experiment guide will initially propose them to confirm this with a simple physical exercise. This consists in performing a series of squats, jumps, or other exercises, where the student should pay attention to his/her heartbeat and respiration rate, and how its frequency will increase along time. This initial stage will enable the students to confirm their knowledge about physical activities and the various measured signals.

After this simple introduction, the remaining is devoted to immerse the student in different situations, each of them with the purpose of inducing specific sensations, demanding more or less mental workload, and/or induce emotional states. For the classroom use case, three different types of scenarios are proposed, as follows:

Scenario Type A. Objective: to elicit fear and anxiety. The user will be asked to search for a specific object in a poorly lit and grim haunted house where, like in any horror movie, ghosts can suddenly appear out of nowhere. While experiencing this scenario the user will not only feel fear but also will exhibit escape reactions (Figure 1, Up Left). 
Scenario Type B: Objective: to elicit peace/calmness. The user is in the middle of a virtual Zen garden with verdant plants and natural environment objects. The whole imagery and sound related to this experience should induce peaceful and calming sensations and feelings (Figure 1, Up Right).

Scenario Type C. Objective: to elicit momentary activations. The user will have to deal with harmful events to his/her virtual representation. All of these unexpected and frightening situations should make the user feel anxious, stressed and life threatened. Once again, this is only possible to safely execute through the use of virtual reality (Figure 1, Down).

\section{B. Experimental Apparatus}

The setup needed for this experiment requires three different devices: a laptop, an Oculus Rift and a BITalino. The laptop doesn't require any exceptional hardware, as the virtual environments are not especially demanding. Any standard and recent laptop should run the software smoothly enough. The Oculus Rift is one of the most advanced head mounted displays and produces a field of view good enough to make the experience adequately immersive. BITalino is a low-cost didactic toolkit especially designed to work with bio-signals. The standard kit comes with most of the sensors needed for this experiment, such as, Electrocardiography (ECG) to measuring the heart rate, Electro Dermal Activity (EDA) to galvanic skin response analysis, and Accelerometers (ACC) to body parts accelerations. Apart form the signals captured by BITalino, other like temperature, or breath rate (BR) can also be analyzed as long as the appropriate sensors are available. Figure 3 illustrates the distribution of the sensors on the user's body, where the head mounted display is represented as its accelerometer, or an additional one attached to it used to register escape or other reactions. The ECG electrodes are placed in the intercostal space at the midclavicular line; the EDA electrodes are attached one to the index finger and the other one to the ring finger; the accelerometer is fixed to the head mounted display; the thermometer is placed at skin surface in the wrist; and finally the BR-band tighten around the chest.

The figure 3 represents a set of possible experience configurations for the experiment procedure, where A represents an activating environment, $\mathrm{B}$ represents a relaxing environment and finally $\mathrm{C}$ is a single stimulus environment. The red line shows the tendency of the internal activation of the person. This can be traduced by an increase of the sympathetic nervous system activity in stressful periods $(\mathrm{A}, \mathrm{C})$ or on the other hand a preponderance of the parasympathetic part in the relaxing temporal windows (B). The experimental procedure can then have distinct situations combining opposite environments (first case) to see activation/relaxation transitions or single environments (second and third case).

Figures 4 and 5 show examples of expected results from two experiments, an activating one (type A) and a relaxing one (type $\mathrm{B}$ ), respectively. Both set of signals are normalized in the same scale, as the shapes of the plots are more important for this experiment than the absolute vales of each measured variable. From these shapes, we can easily notice distinct different tendencies in each of of the measured activity or physiological signals.
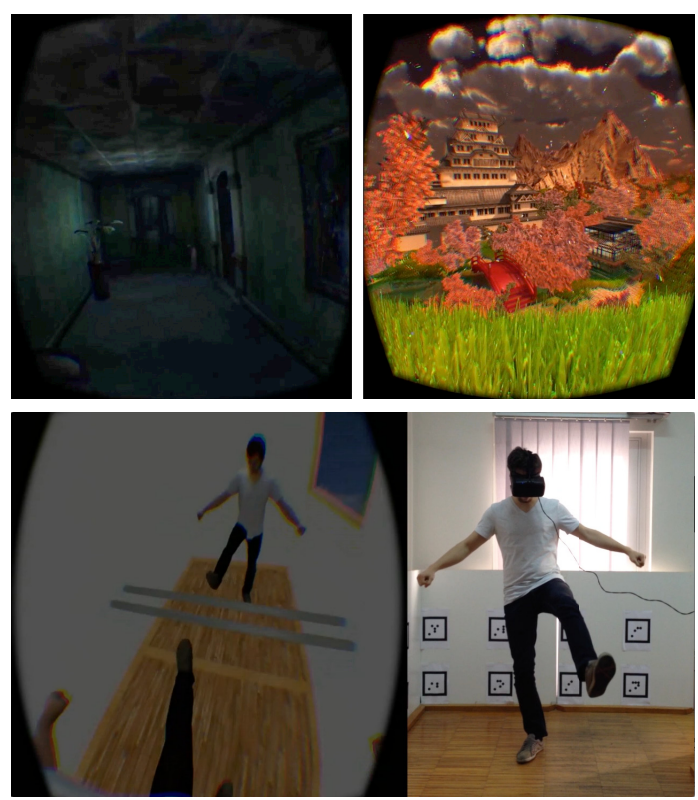

Figure 1. Examples scenario types: Up Left - Type A - Haunted house environment; Up Right - Type B - Zen garden environment; Down -

Type $\mathrm{C}$ - Harmful event to self-virtual representation

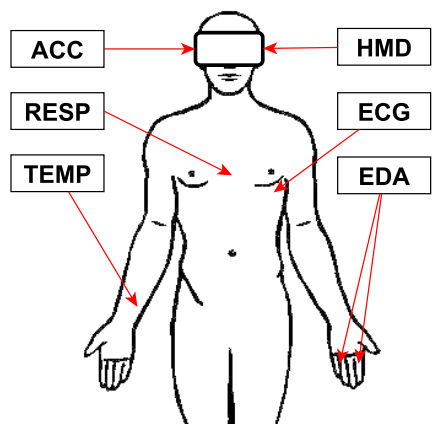

Figure 2. Placement of the sensors and head mounted display.

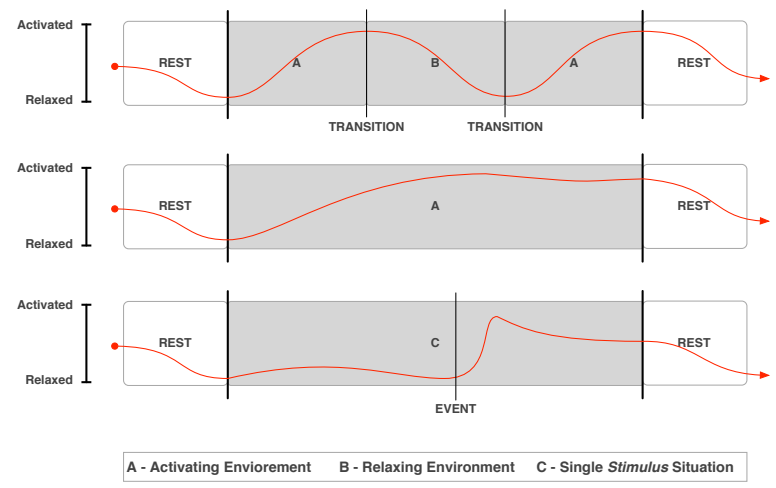

Figure 3. Possible configurations and expected results (red line).

Starting by the Head Movements (cyan line), in an activating environment, a person tends to be in a more alerted state creating more abrupt head movement accelerations, while exploring the surrounding environment.

In terms of EDA (magenta line) a relaxing experiment will tend to have fewer stimuli making the curve line smoother over time, while in a stressful environment, we can easily spot a lot of isolated peaks. 


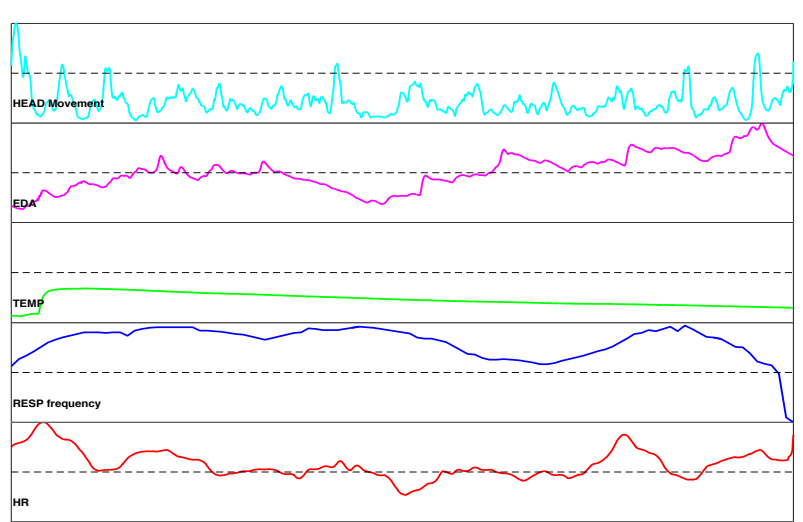

Figure 4. Collected data from type A environment.

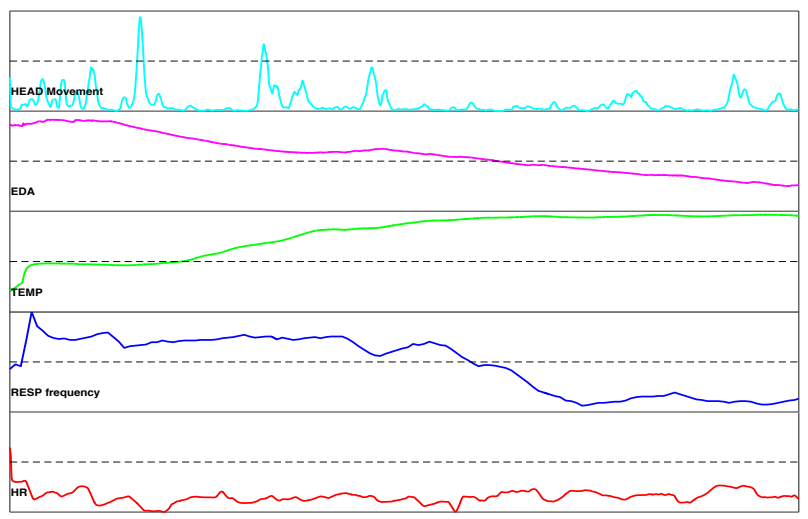

Figure 5. Collected data from type B environment.

Overall, the EDA mean tend to be correlated with the sympathetic activation, rising in stress and decreasing in relaxes. Temperature signal (green line), when measured on the skin surface (e.g. in the wrist) tends to get lower in stress due to increased sweating.

In relaxing environments, as the skin is drier, it has an opposite effect until stabilization. Respiration rate (blue line) can be a good indicator of the internal activation of the person. It can indicate periods of hyperventilation in stress events. It is expected to be higher in activation environments than in relax.

Finality the heart rate (red line) is another good indicator of the person's sympathetic nervous system activity.

In terms of a single stimulus analysis (type C), figure 6 presents an example of a typical EDA response. After the stimulus is presented to the person, a great increase in EDA can normally be observed, after a small time delay.

The above selected scenarios are simple examples to demonstrate the type of software that can be used. The instructor can select other ones to produce similar effects, based on preference or availability.

\section{CONCLUSION}

In this paper we propose the use of immersive systems to teach students about the relations between emotions and physiological states. For attaining this purpose, a VR based system is proposed where three different types of scenarios aim at inducing specific sensations and emotions on the students. The measured heart rate, galvanic skin response, body accelerations, temperature changes, and breath rate are to be analyzed by students and compared with their changes between activities. It is expectable that

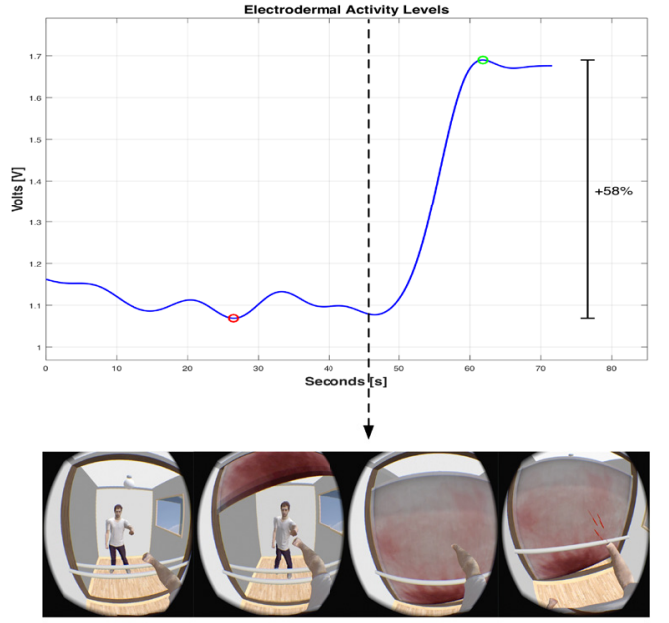

Figure 6. Harmful event example (bottom) and its relation with physiological data (top).

at the end of the proposed laboratory experiment, the student will be able to establish a new relationship of these parameters and better understand their manifestations on his/her own body.

\section{REFERENCES}

[1] D. Purves, G. J. Augustine, D. Fitzpatrick, et al., editors. Neuroscience. 2nd edition., Sunderland (MA): Sinauer Associates, 2001.

[2] R. Cowie, E. Douglas-Cowie, N. Tsapatsoulis, G. Votsis, S. Kollias, W. Fellenz, J. G. Taylor, Emotion recognition in humancomputer interaction, Signal Processing Magazine, IEEE, vol.18, no.1, pp.32-80, Jan 2001. http://dx.doi.org/10.1109/79.911197

[3] S. Craig, A. Graesser, J. Sullins, and B. Gholson, Affect and learning: an exploratory look into the role of affect in learning with AutoTutor. Journal of educational media, 29(3), 241-250, 2004. http://dx.doi.org/10.1080/1358165042000283101

[4] M. Roussou, M. Oliver, and M. Slater, The virtual playground: an educational virtual reality environment for evaluating interactivity and conceptual learning. Virtual reality 10.3-4: 227-240, 2006.

[5] T. Restivo, J. Rodrigues, F. Chouzal, P. Menezes, Online virtual system for straightness evaluation, Experiment@ International Conference (exp.at'13), 2013, 192-193, 2013.

[6] L. Shen, M. Wang and R. Shen. Affective e-Learning: Using Emotional Data to Improve Learning in Pervasive Learning Environment., Educational Technology \& Society, 12 (2), 176-189, 2009.

[7] H. Q. Dinh, et al., Evaluating the importance of multi-sensory input on memory and the sense of presence in virtual environments. Virtual Reality, 1999. http://dx.doi.org/10.1109/vr. 1999.756955

[8] G. Riva, et al., Affective interactions using virtual reality: the link between presence and emotions. CyberPsychology \& Behavior 10.1: 45- 56, 2007. http://dx.doi.org/10.1089/cpb.2006.9993

\section{AUTHORS}

Bruno Patrão is with the Institute of Systems and Robotics, University of Coimbra, Portugal (e-mail: bpatrao@isr.uc.pt).

Samuel Pedro is with the Institute of Systems and Robotics, University of Coimbra, Portugal (e-mail: samuel@isr.uc.pt).

Paulo Menezes is with the Institute of Systems and Robotic, Electrical Engineering and Computer Department, University of Coimbra, Portugal, (e-mail: pm@deec.uc.pt).

Submitted 27 September 2015. Published as resubmitted by the authors 13 December 2015. 